\title{
ROUTING PROTOCOL BASED ON ENERGY EFFICIENT FOR UNDERWATER WIRELESS NETWORK
}

\author{
Pradeep $S^{1}$, Tapas Bapu B $R^{2}$, Maheswaran $U^{3}$, Thirunavukkarasan $M^{4}$, Partheeban $N^{5}$, Nagaraju $V^{6}$ \\ ${ }^{1}$ Associate Professor, Department of Electronics and Communication, S.A.Engineering College, Chennai, Tamilnadu, 600077. \\ Professor, Department of Electronics and Communication, S.A.Engineering College, Chennai, Tamilnadu, 600077 \\ Asssistant Professor, Department of Electronics and Communication, Rajalakshmi Institute of Technology, Chennai, Tamilnadu \\ ${ }^{4}$ Assistant Professor, School of Computing Science and Engineering, Galgotias University, Uttar Pradesh, India \\ ${ }^{5}$ Professor, School of Computing Science and Engineering, Galgotias University, Uttar Pradesh, India, \\ Professor \& Head, Department of Electronics and Communication, Rajalakshmi Institute of Technology, Chennai, Tamilnadu
}

DOI: $10.51201 / J U S S T / 21 / 05195$

http://doi.org/10.51201/JUSST/21/05195

\begin{abstract}
Underwater Wireless Sensor Networks is an effective and intelligent utilization of energy for routing protocol in longer network lifetime. Energy Consumption and load balancing are the vital role for network life time. The uses of load balancing in WSN is granted as best resources of sink mobility which protects energy sources to organize. The aim of this paper is to evaluate various deployed strategies involving sink mobility. Multiple mobile sinks are capable of performing computational operations like collecting information from electric joints instantly, storage and also communication capability. It evaluates the results and the effect of sink mobility by comparing with another routing protocol GEDAR.
\end{abstract}

Index Terms - routing protocol, invalid connection, uses of power, computational operations, electric joints

\section{INTRODUCTION}

WSN plays a major role in all fields because of their vast application. This network is worldly WSN used for various place of nature and transmission of data (Akyildiz et al., 2005) The sensing devices of network activate many applications like detection of deep water, observation of water level, calamities under water, discovery of lubricate items under water and finding mineral materials in far deep places in a consort manner. (Vasilescu et al., 2005) UWSN have many unique characteristics such as battery powered sensor nodes and low frequency. These features of UWSN guide to many problems like no battery replacement and no applicable battery recharging. (Xu et al., 2004) In Underwater Wireless Sensor Network constitute many challenges like giving information much late, low sound speed of $2342 \mathrm{~m}$, received a little amount of a data strea $\mathrm{m}$, low frequency, two or more ways for receiving signal and so on (Paek et al., 2005) To solve this network lifespan, many routing protocols are proposed. (Szewczyk et al., 2004) The simple and extensible mode are accepted by geographical routing energy method. (Climent et al., 2014) This method faces battery issues

i. e nodes need to be charged at regular intervals because the battery life of the nodes is very low. (Coutinho et al., 2015a) The exhaustion of battery nodes creates energy hole. This hole node failed to communicate with other nodes in the network. Because of this failure a node cannot transfer data to a final node. (Coutinho et al., 2015b) A prototype called geoopportunistic routing lighten the hidden problems in nodes. (Coutinho et al., 2017) Sink Mobility aims to collect information instantly from electric joints and retrieve information from invalid joint. (Liu et al., 2016). The purpose of sink mobility is to find the minerals from the deep ocean where humans are not to detect. 


\section{PROPOSED PROTOCOL}

\section{A. WEB CONSTRUCTION}

As for three-dimensional web construction, we focus two major things they are homogeneous network and sinks which are stable at the water surface. More than three sinks and nodes are placed underwater to transfer data successfully for communication range. All these underwater devices have finite amount of space, connection for sound services, receiving information and storage-cell. Some well positioned underwater networks have both sound channelized and wireless data trans- mission. Underwater network connection and static descend plays a major role. The important work for web construction is to rectify data from deep places under water and must be steadfast to give information to the extraterritorial places.

\section{B. Aspects of Neighbour Joint in WSN:}

This selection node phase or two or more joints could develop rising data to prospective data and forward data to the underwater joints. This sending method based on systematic node and it finds the depth and distance for forwarding node and also used to drop or choose the feasible joint for distance. In this phase, receiving nodes establishes its own factor to compare the value which is received in the data packet. Higher value nodes become the eligible nodes for forwarding the information

\section{Stage of forwarding and routing the data:}

This topic focuses on the problems of routing protocols which are based on data forwarding. These nodes forward their packets in shorter range of surface where the joints start by itself. Here the load balancing is constant to the high frequency of underwater network. While transferring data, if a joint is unable to find sinks, then it will have the greedy approach of multi-hop mechanism. Suppose if a joint is captured in a communication trap, then it does not find high flexible joint or neighbor underwater network to collect or forward data. This capture joint transmits a invalid joint to the neighbor sink to ignore the losing of data and communication net. Communication protects the life of cell which is in underwater and accept the web joints to work for a certain time. So, transmission allows data for both deliver and received method for further processing. The void node is activated to change its progress when the mobile sinks receive the message from void declaration. The invalid joint transfers the information to the neighbor joint when network sensor circulates its transmission range. And finally, radio link is used to monitor the centre of the surface sinks which transmits data.

\section{Balancing of Load in WSN:}

Load balancing desire to gather wireless sensor reading from the fields of sensory at defined underwater for analysis and processing. It is used to analyze the consequence of load balancing when invalid network occurs. When it operates to forward, suddenly a joint capture by invalid region and unable finds its way to proceed the network information for both its own region and neighbor information. Void node operates to stop this greedy forwarding 
method for collecting data. An invalid joint of packet is not only notify load balance but also to low depth neighbors node in underwater wireless sensor network. When neighbor node receives its message, it soon sends declaration message to void node with its area and its own message (neighbor). This method is called recovery of message. The downstream joint and void node jointly less trapped in the invalid nodes. Therefore, all packets of data cannot be able to modify the transmitted data packet when it's dropped all potential forwarders. To overcome this trap, uniform mobile sink circulation must be performed. The dimension contains three network area are used to retrieve information which is in invalid place. $\mathrm{X}, \mathrm{Y}, \mathrm{Z}$ nodes coordinated when collecting invalid node points.

\section{E. Algorithm of MSEERP}

MS - Mobile sink; MS_RC - Transmission range of MS; ne-neighbor node of $M S$

$\mathrm{Nr}$ - neighbor node of ne; $\mathrm{Ne} \_$rc - Transmission range of ne

vn: void node

vn_nmsg: void_node_notification_message

Step 1: MS broadcasts hello packet periodically and waits for pause time

Step 2: ne receives the hello packet

Step 3: if dis (ne, MS) <ne_rc)

Step 4: ne sends data to $\overline{\mathrm{MS}}$

Step 5: if (vn_nmsg is recd by MS)

MS changes its position

Ms broadcasts hello packet

vn sends data to MS

MS travels back to prev.loc

go to step 1

else

MS remains till pause time.

Repeat step 4 with next neighbor

end if

else

Step 6: ne broadcasts packet to $\mathrm{Nr}$

Step 7: Nr with highest RDF value $\rightarrow>$ forwarder Step ne $->\mathrm{Nr}$

go to step 3 end if

\section{ANAL YSIS OF SIMULATION:}

The proposed work of simulation results which carried out its replica in WaterSimulation (Yan et al., 2018) are existing against the network WW-SD (Han et al., 2019). This method is assessed by three methods, they are

- PDR

- One packet and node for energy consumption

- E to E delay

Some of the following methods which highlights these results are 


\section{A. The Parameters of Simulation:}

The activation of simulation joints is differing from 200 to 450 with 56sono buoys placed at the deep surface of water and 6 underwater networks to collect information from mobile sinks. The dimensions of networks are $1800 \mathrm{~m}$ x1800 m x $1800 \mathrm{~m}$. Moreover, the range of transmission is $250 \mathrm{~m}$ and Data rate $=65 \mathrm{kbps}$ Tables I and II.

Table.1 parameters of simulation

\begin{tabular}{|l|c|}
\hline \multicolumn{1}{|c|}{ Parameter } & Value \\
\hline Network size & 1500x1500x1500 \\
\hline Sensor nodes & $100-500$ (in steps of 50) \\
\hline Mobile sinks & 10 \\
\hline Static Surface Sinks & 45 \\
\hline Transmission Power & $2 \mathrm{~W}$ \\
\hline Reception Power & $0.1 \mathrm{~W}$ \\
\hline Idle Power & $10 \mathrm{~m} \mathrm{~W}$ \\
\hline Mobility Model & Random way Point \\
\hline Threshold Energy & $10 \mathrm{~J}$ \\
\hline Data rate & $10 \mathrm{kbps}$ \\
\hline
\end{tabular}

Table 2 Node Vs Mobile Sink

\begin{tabular}{|c|c|c|}
\hline & Node & Mobile sink \\
\hline Initial Energy & $100 \mathrm{~J}$ & $300 \mathrm{~J}$ \\
\hline Transmission range & $250 \mathrm{~m}$ & $1000 \mathrm{~m}$ \\
\hline
\end{tabular}

DP size is 130 with a payload of 190 bytes in each dp and 35 bytes of signal data. The dissipation of energy associated with communication, reception, constant state and the adjustment of depth is $\mathrm{Pd}=7 \mathrm{~W}, \mathrm{Ps}=0.8 \mathrm{Wand} \mathrm{Pi}=176 \mathrm{Wm}$ respectively. The average optimal results of 50 distinctive simulation was plotted in the graphs. The parameters of simulation are marked in Table I .

\section{PACKET DELIVERY RATIO (PDR)}

PDR can be valued by the ratio of total delivered packets to the no. of packets totally send from origin mode in the network. When the value of Packet Delivery Ratio increases automatically the network performance also increased. A node is attacked by hole which is available in network that shows a location of mobile sinks. An increasing level of PDR is depicted in Figure 1 


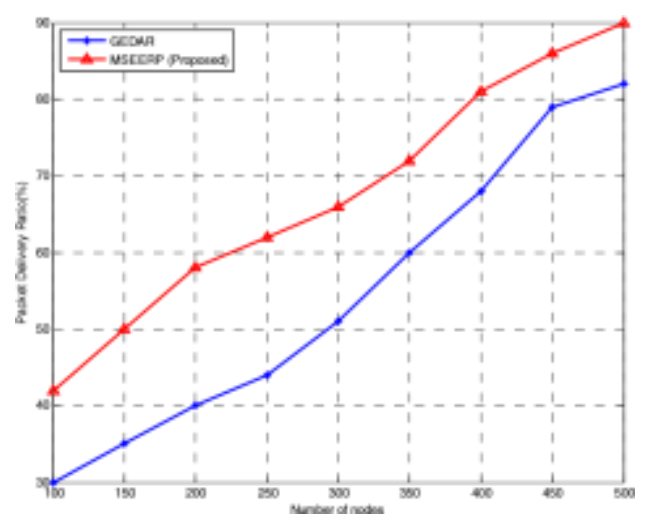

Figure 1 Packet Delivery Ratio

\section{ENHANCEMENT OF ENERGY}

The enhancement of energy consumption is reduced because when neighbor nodes find various nodes for communication. The mobile sinks look after the acoustic environment when energy consumption is related to a distance and at the same time the nodes also find neighbors for communication. During that period, the energy level is decreased. Because of depth adjustment only the energy level increased in GEDAR wireless sensor networks. This energy utilization is clearly in the plotted graph 2.

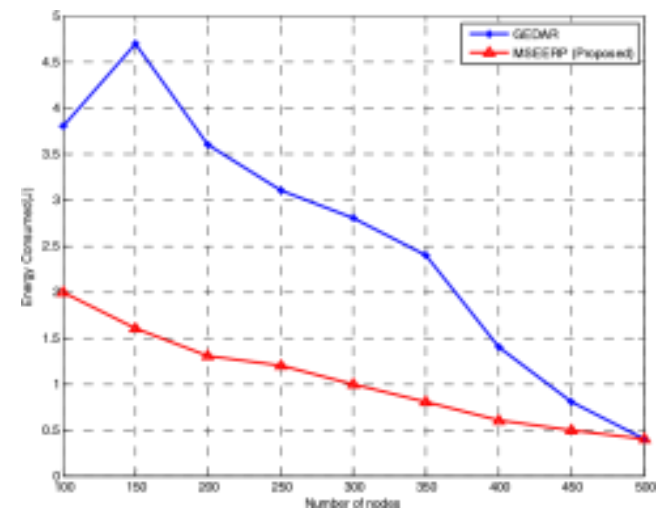

Figure 2 Enhancement of Energy

\section{A. E To E Delay}

E to E delay consists many joints to participate in transmission when the thickness of joint is in high level. An alleviation of re-transmission is not only the reason for less delay but also for the scheme and selection of high-quality link. The no. of bound taken to send information to underwater network is less slightly varied to other methods for collecting information from direct audible joints the E-to-E delay is clearly shown in figure 3. 


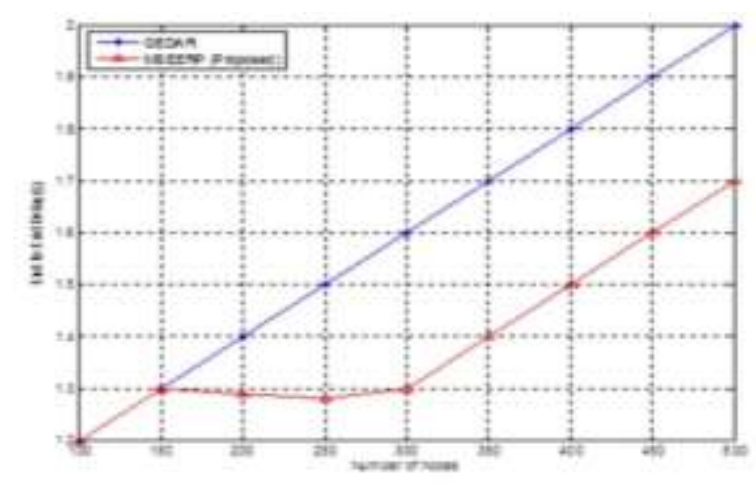

Figure 3 End to End Delay

\section{CONCLUSION}

In this paper, how routing protocol based on energy efficient in the wireless sensor protocol and sink mobility are explained and investigated in a wide manner through different examples, types and procedures. The disseminated protocol are proposed to neighbor load balancing directly from a photoelectric joint which is used to find load balancing during transmission time or it changes the joints in a multiple bounded areas to find a load balancing. $\mathrm{E}$ to $\mathrm{E}$ intermission and information transmission level are improved when information is received straight to the load balancing and uses of power of energy and web construction time were developed when information is received. The load balancing also clear the invalid transmission problem while gather the information from invalid joint to acquire any cleared methods.

\section{REFERENCES}

[1] I. F. Akyildiz, D. Pompili, and T. Melodia, "Underwater acoustic sensor networks: research challenges," Ad Hoc Networks, vol. 3, no. 3, pp. 257-279, 2005. [Online]. Available: 10.1016/j.adhoc.2005.01.004; https: //dx.doi.org / 10.1016 / j.adhoc. 2005. 01.004

[2] Vasilescu and Iuliu, "Data collection, storage, and retrieval with an underwater sensor network," Proceedings of the 3rd international conference on Embedded networked sensor systems, 2005.

[3] N. Xu, "A wireless sensor network for structural monitoring," Proceedings of the 2nd international conference on Embedded networked sensor systems, 2004.

[4] J. Paek, "A wireless sensor network for structural health monitoring: Performance and experience," The Second IEEE Workshop on Embedded Networked Sensors, 2005.

[5] R. Szewczyk, "An analysis of a large scale habitat monitoring appli- cation," Proceedings of the 2nd international conference on Embedded networked sensor systems, 2004.

[6] S. Climent, A. Sanchez, J. Capella, N. Meratnia, and J. Serrano, "Underwater Acoustic Wireless Sensor Networks: Advances and Future Trends in Physical, MAC 
and Routing Layers," Sensors, vol. 14, no. 1, pp. 795-833, 2014. [Online]. Available: 10.3390/s140100795;https://dx.doi.org/10.3390/s140100795

[7] R. W. Coutinho, "A novel void node recovery paradigm for long-term underwater sensor networks," Ad Hoc Networks, vol. 34, pp. 144-156, 2015.

[8] "Geographic and opportunistic routing for underwater sensor networks," IEEE Transactions on Computers, vol. 65, pp. 548-561, 2015.

[9] "EnOR: Energy balancing routing protocol for underwater sensor networks," 2017 IEEE International Conference on Communications (ICC), 2017.

[10] M. Liu, "On-surface wireless-assisted opportunistic routing for under- water sensor networks," Proceedings of the 11th ACM International Conference on Underwater Networks \& Systems, 2016.

[11] J. J. Kartha and L. Jacob, "Delay and Lifetime Performance of Underwater Wireless Sensor Networks with Mobile Element Based Data Collection," International Journal of Distributed Sensor Networks, vol. 11, no. 5, pp. 128 757-128 757, 2015. [Online]. Available:10.1155/2015/128757;https://dx.doi.org/10.1155/2015/128757

[12] T. Wang, "A survey on geographic routing protocols in delay/disruption tolerant networks," International Journal of Distributed Sensor Net- works, vol. 12, pp. 3174 670-3 174 670, 2016.

[13] J. Yan, "Energy-efficient data collection over AUV-assisted underwater acoustic sensor network," IEEE Systems Journal, vol. 12, pp. 3519-3530, 2018.

[14] G. Han, "An AUV location prediction-based data collection scheme for underwater wireless sensor networks," IEEE Transactions on Vehicular Technology, vol. 68, pp. 6037-6049, 2019.

[15] Jorge and A. M. Vitor, "A survey on unmanned surface vehicles for disaster robotics: Main challenges and directions," Sensors, vol. 19, pp. 702-702, 2019.

[16] N. Javaid, "Cooperative opportunistic pressure based routing for under- water wireless sensor networks," Sensors, vol. 17, pp. 629-629, 2017.

[17] R. W. Coutinho, "GEDAR: geographic and opportunistic routing pro- tocol with depth adjustment for mobile underwater sensor networks," IEEE International Conference on communications (ICC), 2014.

[18] W. Zhuo, "AUV-aided communication method for underwater mobile sensor network," OCEANS 2016-Shanghai, 2016. 